\title{
La relación entre factores de la personalidad y conductas y cogniciones sexuales: un estudio correlacional
}

\author{
Jiménez Rodríguez, Ángela \\ Universidad Católica Santa María la Antigua, Panamá \\ atjimenezr@usma.com.pa \\ Álvarez, Sofía \\ Universidad Católica Santa María la Antigua, Panamá
}

\begin{abstract}
Personality is defined as a pattern of relatively permanent traits and unique characteristics that confer coherence and individuality to a person's behavior (Feist, 2014). David Schmitt (2011) differentiates the "social" personality from the one that appears at sexual situations. This classification is based on the behavioral differences that can be observed between the sexual and social life of certain people. The following research question was raised: What personality factors influence sexual cognitions and behaviors? The population used was university students of Panama, of between 18 and 25 years, who had had sexual relations. Among the characteristicsof the research participants, it was found that their mean age was 20.65 (SD = 1.78) and around65\% were male. 60\% identified as heterosexual. Of the total sample, $65 \%$ were currently havingsexual intercourse. The results show that introverts have a higher level of exploratory cognitionsthan extroverts; those who currently have sex have on average a higher level of sadomasochisticcognitions; people who are in relationships on average have more exploratory, impersonal cognitions, and intimate behaviors. Due to the results, it could be observed that the social personality and the sexual personality do not present clear correlations and that the sexual one fluctuates in the sexual spheres. Additionally, data was obtained that had not been observed in similar research, such as the negative relationship between cognitive reserve and sexual cognitions and behaviors.
\end{abstract}

Keywords: personality, sexuality, sexual cognition, sexual behavior, factors. 


\section{RESUMEN}

La personalidad se define como un patrón de rasgos relativamente permanentes y de características singulares que confieren coherencia e individualidad al comportamiento de una persona (Feist, 2014). David Schmitt (2011) diferencia este tipo de personalidad de las que se dan en situaciones sexuales. Esta clasificación se basa en las diferencias comportamentales quese pueden observar entre la vida sexual y social de ciertas personas. Se planteó la siguiente pregunta de investigación: ¿Qué factores de la personalidad influyen en las cogniciones y conductas sexuales?. La población fueron universitarios residentes de Panamá, entre los 18 y 25 años de edad, que hubieran tenido relaciones sexuales. Entre las características de los participantes de la investigación, se encontró que la edad media de estos fue de 20.65 (DE=1.78) y alrededor del 65\% eran varones. El $60 \%$ se identificaron como heterosexuales. De la muestra total, el $65 \%$ se encontraban manteniendo relaciones sexuales actualmente. Los resultados muestran que los introvertidos tienen un mayor nivel de cogniciones exploratorias que los extrovertidos; los que actualmente mantienen relaciones sexuales tienen en promedio un mayornivel de cogniciones sadomasoquistas; las personas que están en relaciones en promedio tienen más cogniciones exploratorias, impersonales y conductas íntimas. Debido a los resultados, se pudo observar que la personalidad social y la personalidad sexual no presentan correlaciones claras y que la sexual fluctúa en los ámbitos sexuales. Adicional, se obtuvieron datos que no habían sido observados en investigaciones similares, como la relación negativa entre la reserva cognitiva con las cogniciones y conductas sexuales.

Palabras claves: personalidad, sexualidad, cognición sexual, conducta sexual, factores.

\section{INTRODUCCIÓN}

La personalidad es un patrón de conducta y pensamiento que se mantiene relativamente estable en diferentes situaciones y a lo largo de la vida [1]. Esta es la manera en la que nos presentamos y como el resto del mundo nos conoce y en varias investigaciones se trata de comprobar si estos rasgos de la personalidad son un patrón estable en distintas situaciones. Delas muchas teorías sobre la personalidad, se utilizó en esta investigación la propuesta por Raymond Cattell (1973), la cual define a la personalidad como compuesta por rasgos comunes, presentes en muchos individuos, rasgos singulares, específicos de cada individuo y por rasgos fuentes, capaces de predecir o explicar una conducta que co-varía [1]. 
Se han realizado estudios que juntan las variables de personalidad y sexualidad, sin embargo, son pocos los que hablan de la relación entre los rasgos de la personalidad y el aspectocognitivo (fantasías y pensamientos sexuales) y conductual de la sexualidad. Las conductas sexuales son todo tipo de práctica sexual que una persona puede realizar consigo misma, con otra persona o en grupo y las cogniciones sexuales son todo tipo de pensamiento con algún contenido sexual [2]. Algunos ejemplos de estos son los realizados por Sierra, Alvarez-Castro yMiró [3], en el cual se encontró que los introvertidos presentan más cogniciones sexuales correspondientes a la intimidad, Renaud y Byers [4] crean los términos de cognición sexual y cognición sexual positiva y negativa, Moyano y Sierra [3] correlaciona los rasgos de personalidady las cogniciones sexuales positivas y negativas; y Heaven et al [5], el cual habla sobre la correlación existente entre la personalidad y la conducta sexual.

No se han realizado estudios previos, ni en Panamá ni en otra parte del mundo, que explorenla relación de los factores de la personalidad y las cogniciones y conductas sexuales. Considerando la ausencia de investigaciones previas que puedan guiar nuevas hipótesis, no se delinearon predicciones específicas con respecto a la relación entre las variables sociodemográficas, los rasgos de personalidad y las cogniciones y conductas sexuales. En cambio, se planteó la siguiente pregunta de investigación: ¿Qué factores de la personalidad influyen en las cogniciones y conductas sexuales?

\section{MÉTODO}

\section{A. MÉTODO Y MUESTRA}

Esta investigación fue transversal y aplicada, de tipo cuantitativo, descriptivo, correlacional yno experimental. La población utilizada fueron personas adultas, residentes de la República de Panamá, entre los 18 y 25 años, que asisten a la universidad y que han tenido relaciones sexuales. La muestra fueron 20 personas. Se realizó un muestreo por conveniencia, según la accesibilidad y proximidad de los sujetos.

\section{B. HERRAMIENTAS UTILIZADAS}

- Se aplicó una entrevista sociodemográfica semiabierta, que permitió a las investigadorasclasificar a los participantes y obtener datos importantes para realizar conclusiones.

- El Test de Personalidad de los 16 factores de Cattell que buscó identificar el tipo y rasgosde personalidad que el participante tiene. Se aplicó de manera presencial en unacomputadora o de manera virtual enviando el link. [6]

- El Sexual Cognition Checklist, de Byers y Renauld, que consta de 56 ítems de cogniciones 
sexuales. Se le pidió al participante reportar la frecuencia y el tipo de pensamiento que tienen sobre aspectos sexuales. [7]

La Entrevista de Conductas Sexuales, adaptada del SCC de Byers y Renauld por las investigadoras, buscó que el participante identificara las conductas sexuales que practica.

\section{RESULTADOS}

\begin{tabular}{|c|c|}
\hline Variable & $\begin{array}{c}\text { Muestra } \\
(n=20) \\
\text { Media (DE) ó \# (\%) }\end{array}$ \\
\hline \multicolumn{2}{|l|}{ Sexo } \\
\hline Masculino & $13(65)$ \\
\hline Femenino & $7(35)$ \\
\hline Edad & $20,65(1,78)$ \\
\hline \multicolumn{2}{|l|}{ Nacionalidad } \\
\hline Panameño & $15(75)$ \\
\hline Extranjero & $5(25)$ \\
\hline \multicolumn{2}{|l|}{ Estado civil } \\
\hline Soltero & $14(70)$ \\
\hline En relación & $6(30)$ \\
\hline \multicolumn{2}{|l|}{ Orientación sexual } \\
\hline Heterosexual & $12(60)$ \\
\hline Homosexual & $2(10)$ \\
\hline Bisexual & $5(25)$ \\
\hline Pansexual & $1(5)$ \\
\hline \multicolumn{2}{|l|}{ Nivel de educación } \\
\hline Licenciatura & $19(95)$ \\
\hline Maestría & $1(5)$ \\
\hline \multicolumn{2}{|l|}{ Estudia actualmente } \\
\hline Sí & $19(95)$ \\
\hline No & $1(5)$ \\
\hline \multicolumn{2}{|l|}{ Facultad } \\
\hline Humanidades y Teologia & $1(5)$ \\
\hline Negocios & $7(35)$ \\
\hline Ingeniería y Tec nología & $2(10)$ \\
\hline Derecho y Ciencias Políticas & $3(15)$ \\
\hline Arquitec tura y Diseño & $2(10)$ \\
\hline Ciencias Social & $5(25)$ \\
\hline \multicolumn{2}{|l|}{ Trabaja actualmente } \\
\hline Sí & $7(35)$ \\
\hline No & $13(65)$ \\
\hline \multicolumn{2}{|l|}{ Relaciones sexuales } \\
\hline Actualmente & $13(65)$ \\
\hline En el pasado & $7(35)$ \\
\hline
\end{tabular}

Se realizaron análisis de correlación y pruebas t de Student para determinar si existía una relación entre las variables.

Al observar las correlaciones entre los 16 factores de la personalidad y las cogniciones y conductas sexuales, resumidas en la tabla 2.1, resultaron múltiples correlaciones significativas con un $\mathrm{p}$ superior a .01. Las cogniciones íntimas se correlacionaron positivamente con complejidad y curiosidad intelectuales. Las cogniciones exploratorias se correlacionaron negativamente con reserva cognitiva y positivamente con complejidad intelectual. Las cogniciones sadomasoquistas se correlacionaron positivamente con asertividad y empatía y negativamente con reserva cognitiva. Las cogniciones impersonales se correlacionaron positivamente con imaginación y amigabilidad y negativamente con reserva cognitiva. Las 
conductas impersonales se correlacionaron negativamente con reserva cognitiva y positivamente con amigabilidad.

Las cogniciones y conductas sexuales comparadas consigo mismas también arrojaron correlaciones significativas, todas de ellas siendo positivas. Las cogniciones íntimas se correlacionaron con las cogniciones exploratorias, las cogniciones impersonales y las conductas íntimas. Las cogniciones exploratorias se correlacionaron con las cogniciones sadomasoquistas e impersonales. Las

cogniciones impersonales también se correlacionan con las cogniciones sadomasoquistas, además de correlacionarse con las conductas impersonales. Por último, las conductas íntimas se correlacionan con las conductas exploratorias.

\begin{tabular}{|c|c|c|c|c|c|c|c|c|}
\hline & $\begin{array}{l}\text { Indice Cog. } \\
\text { Intimas }\end{array}$ & $\begin{array}{l}\text { Indice } \mathrm{Cog} . \\
\text { Exploratorias }\end{array}$ & $\begin{array}{l}\text { Indice } \mathrm{Cog} . \\
\text { Sadomasoquistas }\end{array}$ & $\begin{array}{l}\text { Indice Cog. } \\
\text { Impersonales }\end{array}$ & $\begin{array}{l}\text { Indice Cond. } \\
5 \text { Intimas }\end{array}$ & $\begin{array}{l}\text { Indice Cond. } \\
\text { Exploratorias }\end{array}$ & $\begin{array}{l}\text { Indice Cond. } \\
\text { Sadomasoquistas }\end{array}$ & $\begin{array}{l}\text { Indice Cond. } \\
\text { Impersonales }\end{array}$ \\
\hline Aser fuidad y Empafa & .338 & .389 & $.575^{*}$ & .293 & .239 & .196 & .303 & $.490^{\circ}$ \\
\hline Sensibilidad &, 135 &,- 016 & -323 &,- 258 & .140 & .086 &,- 011 & -.429 \\
\hline Estabilidad Emocional &,- 131 &,- 159 &,- 054 & .182 &,- 094 & .179 &,- 076 & .154 \\
\hline Afectividad & .066 &,- 023 & -342 & .133 & .285 & .197 & .007 &.,- 185 \\
\hline Imaginación & $.475^{\circ}$ & .411 & .349 & $.684^{-*}$ & .316 & .132 &,- 368 & $.528^{\circ}$ \\
\hline Reser va Cognif va & $-.540^{\circ}$ & $-.687^{*}$ & $-.571^{\cdots}$ &,$- 680^{\circ-}$ & -.267 &,- 343 & .025 & $-.585^{-}$ \\
\hline Obediencia & -352 & -.282 & -375 &,- 191 &,- 385 & -.409 & .069 & -397 \\
\hline Introversión & .039 & -384 & -.284 & .114 &,- 187 & .030 &,- 214 & .156 \\
\hline Control Emocional &, 275 & .056 & .054 &,- 156 & .200 & .071 &,- 010 & -.255 \\
\hline Ansiedad Social & .302 & .212 & .228 & .018 & .142 &,- 124 &,- 038 &,- 198 \\
\hline Complejidad Intelectual & $.563^{*}$ & $.587^{*-}$ & .369 & $.537^{*}$ & $.487^{*}$ & .354 & .288 & .289 \\
\hline Amigabilidad & .386 & $.550^{\circ}$ & .425 & $.773^{*}$ & .268 & .338 & .028 & $.603^{\cdots}$ \\
\hline Gregarismo & .286 & .389 & .112 & .447 & .141 & .280 &,- 020 & .103 \\
\hline Orden y Limpieza & .203 & .221 & .253 & .139 &,- 141 &,- 015 & .228 & .005 \\
\hline Curiosidad Intelectual & $.566^{*}$ & .388 & .358 & .307 & .322 & .130 & .125 & $.459^{\circ}$ \\
\hline Desconfianza & .248 & .097 & 218 & -249 & -.049 & -.086 & 237 & -.044 \\
\hline
\end{tabular}

\begin{tabular}{|c|c|c|c|c|}
\hline Variable & $\begin{array}{c}\text { Soltero } \\
(\mathrm{n}=14) \\
\text { Media (DE) }\end{array}$ & $\begin{array}{l}\text { En relación } \\
\qquad(\mathrm{n}=6) \\
\text { Media (DE) }\end{array}$ & $t$ & $p$ \\
\hline $\begin{array}{l}\text { Îndice Cog. } \\
\text { Întimas }\end{array}$ & $8,12(1,53)$ & $9,09(0,82)$ & $-1,445$ & , 183 \\
\hline $\begin{array}{l}\text { Índice Cog. } \\
\text { Exploratorias }\end{array}$ & $4,62(1,68)$ & $6,79(1,04)$ & $-2,900$ & ,310 \\
\hline $\begin{array}{l}\text { Índice Cog. } \\
\text { Sadomasoquistas }\end{array}$ & $3,83(1,77)$ & $5,17(1,36)$ & $-1,637$ &, 599 \\
\hline $\begin{array}{l}\text { Índice Cog. } \\
\text { Impersonales }\end{array}$ & $4,39(1,82)$ & $5,92(1,69)$ & $-1,750$ &, 500 \\
\hline $\begin{array}{l}\text { Îndice Cond. } \\
\text { Întimas }\end{array}$ & $7,51(1,81)$ & $9,07(, 79)$ & $-2,002$ &, 094 \\
\hline $\begin{array}{l}\text { İndice Cond. } \\
\text { Exploratorias }\end{array}$ & $2,59(1,54)$ & $3,48(1,89)$ & $-1,111$ & ,694 \\
\hline $\begin{array}{l}\text { Índice Cond. } \\
\text { Sadomasoquistas }\end{array}$ & $3,49(2,35)$ & $3,8(1,28)$ &,- 305 & 019 \\
\hline $\begin{array}{l}\text { Índice Cond. } \\
\text { Impersonales }\end{array}$ & $3,79(2,44)$ & $5,42(2,92)$ & $-1,294$ & 859 \\
\hline
\end{tabular}




\begin{tabular}{lcccc}
\hline \multicolumn{5}{c}{ Tabla 4. Cogniciones y conductas sexuales comparadas con la } \\
frecuencia de relaciones sexuales.
\end{tabular}

\begin{tabular}{lcccc}
\hline \multicolumn{5}{c}{ Tabla 5. Cogniciones y conductas sexuales comparadas con nivel de } \\
introversión.
\end{tabular}

Los resultados de las pruebas t de Student realizadas, expuestos en las tablas 3, 4 y 5 , se pueden resumir de la siguiente manera: los introvertidos, en promedio, tienen un mayor nivel decogniciones exploratorias que los extrovertidos; los que actualmente mantienen relaciones sexuales tienen en promedio un mayor nivel de cogniciones sadomasoquistas; las personas queestán en relaciones en promedio tienen más cogniciones exploratorias, impersonales y conductas íntimas 


\section{CONCLUSIONES}

Posterior al desarrollo de la investigación y el análisis de los resultados, se puede afirmar que se cumplieron los objetivos específicos de la investigación, por ende, cumpliendo el objetivo principal, el cualbuscaba determinar si la personalidad social y la personalidad sexual eran estables y congruentes entre sí. Como se pudo observar en las tablas anteriores, estas no presentan correlaciones claras, fluctuando dependiendo dela persona, llevándonos a concluir que puede que la personalidad social y la sexual existanindependientes la una de la otra.

Adicional, se obtuvieron datos que no habían sido observados en investigaciones similares, comola relación negativa entre la reserva cognitiva con las cogniciones y conductas sexuales; además, pudiendoobtener data mediante las pruebas $t$ de Student que confirmara que rasgos extrovertidos, como las conductas exploratorias, se correlacionaron positivamente con personas que se asocian con la personalidad introvertida y vice versa.

Es necesario realizar más estudios en base a este tema, con una mayor muestra para así poder obtener resultados concluyentes.

\section{REFERENCIAS}

[1] Feist, J., Feist, G. and Roberts, T. (2014). Teorías de la personalidad. 8th ed. México: Karen Estrada Arraiaga, pp.4, 243.

[2] Schmitt, D. (2019). What Is Sexual Personality?. [online] Psychology Today. Available at: https:// www.psychologytoday.com/intl/blog/sexual-personalities/201106/what-is-sexual-personality?eml.

[3] Moyano, N., \& Sierra, J. C. (2013). Relationships between personality traits and positive/negative sexual cognitions. International Journal of Clinical and Health Psychology, 13(3), 189-196. https:// doi.org/10.1016/S1697-2600(13)70023-1

[4] Renaud, C. A., \& Byers, E. S. (2001). Positive and negative sexual cognitions: Subjective experience and relationships to sexual adjustment. Journal of Sex Research, 38(3), 252-262. https://doi. org/10.1080/00224490109552094

[5] Heaven, P., Crocker, D., Edwards, B., Preston, N., Ward, R., \& Woodbridge, N. (2002). Personality and Sex. Personality And Individual Differences, 35(3), 411-419. Retrieved from https://www.journals. elsevier.com/personality-and-individual-differences

[6] The Items in the 16 Preliminary IPIP Scales Measuring Constructs Similar to Those in Cattell's 16 Personality Factor Questionnaire (16PF)". <http://ipip.ori.org/new16PFKey.htm>

[7] Renaud, C. A., \& Byers, E. S. (2001). Positive and negative sexual cognitions: Subjective experience and relationships to sexual adjustment. Journal of Sex Research, 38(3), 252-262. https://doi. org/10.1080/00224490109552094 


\section{AUTORIZACIÓN Y LICENCIA CC}

Los autores autorizan a APANAC XVIII a publicar el artículo en las actas de la conferenciaen Acceso Abierto (Open Access) en diversos formatos digitales (PDF, HTML, EPUB) e integrarlos en diversas plataformas online como repositorios y bases de datos bajo la licencia CC: Attribution-NonCommercial-ShareAlike 4.0 International (CC BY-NC-SA 4.0) https://creativecommons.org/licenses/by-nc-sa/4.0/.

Ni APANAC XVIII ni los editores son responsables ni del contenido ni de las implicacionesde lo expresado en el artículo. 